Revue d'histoire de l'Amérique française

\title{
Lettre de J.-J. Girouard à sa femme, 21 mai 1838
}

Volume 19, numéro 3, décembre 1965

URI : https://id.erudit.org/iderudit/302494ar

DOI : https://doi.org/10.7202/302494ar

Aller au sommaire du numéro

Éditeur(s)

Institut d'histoire de l'Amérique française

ISSN

0035-2357 (imprimé)

1492-1383 (numérique)

Découvrir la revue

Citer ce document

(1965). Lettre de J.-J. Girouard à sa femme, 21 mai 1838. Revue d'histoire de

l'Amérique française, 19(3), 463-464. https://doi.org/10.7202/302494ar d'utilisation que vous pouvez consulter en ligne.

https://apropos.erudit.org/fr/usagers/politique-dutilisation/ 


\title{
DOCUMENTS INÉDITS
}

\author{
J.-J. GIROUARD À SA FEMME.
}

Montréal, N. Prison,

21 Mai 1838.

Ma chère amie,

Ma dernière lettre était si longue, si longue qu'elle aurait pu servir pour au moins trois mois si le plaisir et la consolation de m'entretenir avec toi ne me fesaient t'écrire plutôt, persuadé d'ailleurs que je suis de te faire plaisir.

$\mathrm{Tu}$ trouveras ci-joint un tableau des pertes souffertes par les patriotes, que j'ai composé sur des renseignements nombreux et assez exacts que j'ai pu me procurer ici. Ce tableau a été publié sur le Canadien de Québec.

$\mathrm{Tu}$ recevras aussi en même temps un plan de notre prison, auquel M-r Jobin a ajouté le nom des prisonniers qui occupent actuellement les différentes cellules. Cet Edifice a une façade de 224 pieds, et une aîle en arrière de 75 pieds, lui donnant la forme d'un T. Le bâtiment est en pierre de taille à l'extérieur, et a 30 pieds de profondeur sur à peu près autant de hauteur, à l'exception du corps central qui a six pieds de plus en profondeur et est plus élevé que le reste du bâtiment: il contient un attique destiné à servir de chapelle à la prison.

La partie inférieure de cette prison enfoncée d'environ 6 pieds en terre contient les cachots formés de cellules voûtées de $12 \times 8$ pieds, ne recevant de jour que par une espèce de meurtrière pratiqué au haut et n'ayant pas plus de 15 pouces de hauteur sur six pouces de longueur. Le rez-de-chaussée et l'étage au-dessus est rempli de cabannons voûtés de $5 \times 8$ pieds, et où la lumière pénètre par la moitié seulement d'un évantail vitré et grillé en fer ayant environ 12 pouces de rayon. L'étage supérieur qui est celui représenté au plan ci-joint contient trois sales et des chambres de $12 \times 9$ pieds. C'est celui où logent les prisonniers dont tu trouveras les noms marqués sur le plan. Dans l'attique se trouvent une cinquantaine de prisonniers de diverses parties du District. C'est le local destiné à servir de chapelle et où l'on a vu dernièrement entassé jusqu'à 93 prisonniers. Les deux étages inférieures du nôtre sont remplis de prisonniers politiques, et les criminels sont dans les cachots.

Ce bâtiment est un de nos édifices publics les plus mal faits, et c'est en pure perte que la Province y a consacré des sommes immenses. Si au premier abord l'extérieur vous présente quelque chose d'assez régulier, vous n'êtes pas longtemps sans vous convaincre en examinant l'intérieur que les Commissaires chargés de l'économie de cette construction n'avaient pas même l'idée de l'architecture. Rien de plus absurde et de plus malcommode que les distributions de cette prison. Les commissaires voulaient, à ce que j'ai entendu dire à l'un d'eux, faire une prison, un pónitenciaire (sic) et une maison de correction, mais il n'ont fait ni l'un ni l'autre, et tout y est si mal coordonné qu'il faudra, comme on l'a déjà fait y employer annuellement des sommes considérables pour parer aux nombreux inconvéniens qu'un aussi mauvais plan présente continuellement. Ces Messieurs, par exemple avaient été assez stupides que de s'imaginer que l'on pouvait chauffer un édifice comme celui-ci avec de l'air chaud par des tuyeaux circulant et tournant dans les diverses galleries. Point du tout il a fallu nécessairement avoir des poëles, et il n'y a pas une seule place de 
prévue pour les y mettre commodément. A l'exception des pièces du centre destinées aux concierges il n'y a point de cheminées ouvertes dans le reste de la prison, point de ventillateurs dans les très-longues galleries où il n'y a aucun moyen de renouveller l'air. Les privées manquent de cannaux suffisans, se remplissent quelquesfois et répandent l'infection dans la prison. D'ailleurs ces privées sont en trop petit nombre et sont faits sur le plan le plus défectueux. Mais ce qui est le pire c'est que la prison manque d'eau et quelle n'en est pourvue qu'avec beaucoup de négligence, les prisonniers de certaines galleries ayant été quelquesfois jusqu'à 26 heures sans une goutte d'eau, obligé de boire leur eaux sales. On avait adapté une pompe à un puits fait dans la prison où des réservoirs supérieurs répandaient l'eau dans les différentes galleries par des tuyeaux de plomb garnis de chantepleure: ce qui était très-commode. Cependant ces machines étaient si mal construites qu'après avoir coûté en façon et en réparations plusieurs milliers de Louis, il a fallu les abandonner, et se pourvoir d'eau tonne à tonne. On dit que le coût de fournir de l'eau à la prison se monte de cinq à six piastres par jour. Le planchéïage, la menuiserie, vitrerie et toutes les autres parties de cet édifice sont défectueux et déjà dans un état de délabrement à nécessiter des réparations continuelles, et extrêmement coûteuses. Rien, pas la moindre chose dans ce bâtiment qui soit calculé pour les besoins et la commodité des détenus. En un mot, si l'architecture est l'art de construire une édifice dont toutes les parties soient coordonnées et répondent en tous points au but qu'on s'est proposé et à sa destination, assurément les commissaires ont fait tout le contraire.

M. Berthelot est malade au Lit depuis plusieurs jours d'une inflammation dans les reins. Jusqu'à présent toutes les tentatives que nous avons faites pour le faire sortir de prison ont été vaines. On espère pourtant réussir. Emilie est à Montréal, mais elle n'a encore pu voir son père, tant nos tyrans se montrent cruels. M.M. Viger et Cherrier se sont dernièrement adressés au Sheriff pour qu'on leur accordât le droit de voir des avocats pour préparer leurs défenses d'ici. Le Sheriff, pour toute réponse, leur a dit qu'il s'était empressé de faire homologuer par les juges un règlement suspendant ou rescindant le 8 me article des règlements de la prison et par lequel les prisonniers pouvoient voir leurs parens et leurs amis certains jours de la semaine. Cette insolente barberie provoquera des procédés ultérieures de la part de ces messieurs qui vont s'adresser aux Juges pour obtenir justice. Va-t-en voir s'il viennent...

M. Dumouchel et ses enfans sont en parfaite santé et s'amusent assez bien, surtout depuis l'arrivée des prisonniers de la vieille prison.

J'apprends que notre chère petite nièce Henriette est à St Benoit. Si c'est vrai embrasse-la bien étroitement pour moi. Dis-lui de ne pas être trop longtemps à la campagne de peur que ses enfans rebelles qui sont en prison n'en pâtissent.

Amitiés à tous. tout.

Adieu, ma bonne petite femme, courage, patience, et résignation sur

Madame Girouard

J. J. Girouard.

St Benoît.

J'ai écrit si vite et avec une si mauvaise plume que je suis tenté de copier cette lettre si l'occasion me le permet. Tu cours pourtant le risque de l'avoir tel qu'elle est. Lis-là si tu peux. 